OPEN ACCESS

Edited by:

Giuseppe Esposito,

Sapienza University of Rome, Italy

Reviewed by:

Johanna Mahwahwatse Bapela, University of Pretoria, South Africa

Subhash Chandra Mandal, Government of West Bengal, India

*Correspondence:

Nina A. Hering nina.hering@charite.de

Specialty section: This article was submitted to Ethnopharmacology,

a section of the journal

Frontiers in Pharmacology

Received: 25 September 2020 Accepted: 04 February 2021 Published: 10 March 2021

Citation: Hering NA, Luettig J, Jebautzke $B$, Schulzke JD and Rosenthal $R$ (2021) The Punicalagin Metabolites Ellagic Acid and Urolithin A Exert Different Strengthening and Anti-Inflammatory Effects on Tight Junction-Mediated Intestinal Barrier Function In Vitro.

Front. Pharmacol. 12:610164. doi: 10.3389/fphar.2021.610164

\section{The Punicalagin Metabolites Ellagic Acid and Urolithin A Exert Different Strengthening and Anti-Inflammatory Effects on Tight Junction-Mediated Intestinal Barrier Function In Vitro}

\author{
Nina A. Hering ${ }^{1 *}$, Julia Luettig $^{2}$, Britta Jebautzke ${ }^{2}$, Jörg D. Schulzke ${ }^{2}$ and Rita Rosenthal ${ }^{2}$ \\ ${ }^{1}$ Department of General and Visceral Surgery, Charité - Universitätsmedizin Berlin, Berlin, Germasny, ${ }^{2}$ Institute of Clinical \\ Physiology/Nutritional Medicine, Charité - Universitätsmedizin Berlin, Berlin, Germany
}

Scope: Ellagitannins are polyphenols found in numerous fruits, nuts and seeds. The elagitannin punicalagin and its bioactive metabolites ellagic acid and urolithins are discussed to comprise a high potential for therapeutically or preventive medical application such as in intestinal diseases. The present study characterizes effects of punicalagin, ellagic acid and urolithin A on intestinal barrier function in the absence or presence of the proinflammatory cytokine tumor necrosis factor- $\alpha$ (TNFa).

Methods and Results: Transepithelial resistance (TER), fluorescein and ion permeability, tight junction protein expression and signalling pathways were examined in Caco-2 and HT-29/B6 intestinal epithelial cell models. Punicalagin had less or no effects on barrier function in both cell models. Ellagic acid was most effective in ileum-like Caco-2 cells, where it increased TER and reduced fluorescein and sodium permeabilities. This was paralleled by myosin light chain kinase two mediated expression down-regulation of claudin-4, -7 and -15. Urolithin A impeded the TNFa-induced barrier loss by inhibition of claudin-1 and -2 protein expression upregulation and claudin-1 delocalization in HT29/B6.

Conclusion: Ellagic acid and urolithin A affect intestinal barrier function in distinct ways. Ellagic acid acts preventive by strengthening the barrier per se, while urolithin A protects against inflammation-induced barrier dysfunction.

Keywords: barrier function, ellagic acid, punicalagin, tight junction, urolithin A

\footnotetext{
Abbreviations: DAPI, 4',6-Diamidin-2-phenylindol; EA, ellagic acid; ERK1/2; extracellular signal-regulated kinases 1/2; LSM, laser scanning microscopy; MLC2, Myosin Light Chain two; MLCK, myosin light chain kinase; NFkB, nuclear factor 'kappalight-chain-enhancer' of activated B-cells; P, permeability; PCl, chloride permeability; PNa, sodium permeability; Puni, punicalagin; STAT3, signal transducers and activator of transcription 3; TER, transepithelial resistance; TJ, tight junction; TJs, tight junctions; TNF $\alpha$, tumor necrosis factor $\alpha$; UroA, urolithin A; ZO, zonula occludens protein.
} 


\section{INTRODUCTION}

Health benefits of the ellagitannin punicalagin and its metabolites are being extensively discussed in recent years. Punicalagin can be found in pomegranate (Punica granatum), raspberries (Rubus idaeus), strawberries (Fragaria sp.) or walnuts (Juglans regia). Especially pomegranate has already been applied for thousands of years in the traditional medicine (Longtin, 2003) to benefit from its anti-diarrhea (Das et al., 1999; Zhao et al., 2018), anti-oxidant, anti-obesity, anti-cancerogenic, and anti-inflammatory properties (reviewed in Saeed et al., (2018). Ellagitannins are not directly absorbed into the blood stream (Seeram et al., 2004) but hydrolyzed. In the stomach and intestine, hydrolysis of punicalagin yields ellagic acid (EA), which in turn is metabolized to urolithin A and B by the intestinal microbiota (Cerdá et al., 2005; Espín et al., 2007). Several studies showed these metabolites exert anti-inflammatory effects in the intestine. In a mouse model of ulcerative colitis, ellagic acid was effective in reducing intestinal inflammation by inhibiting cyclooxygenase- 2 and inducible nitric oxide synthases. It impeded proinflammatory signaling via nuclear factor 'kappa-light-chainenhancer' of activated B-cells (NFKB) and signal transducers and activator of transcription 3 (STAT3) (Marín et al., 2013). A very recent cell culture study reported ellagic acid to inhibit pro-inflammatory effects of tumor necrosis factor $\alpha$ (TNFa). This involved loss of epithelia barrier function, upregulation of interleukin- 6 and -8 secretion and induction of oxidative stress by impeding $\mathrm{TNF} \alpha$-induced signaling via NF- $\kappa \mathrm{B}$, extracellular signal-regulated kinases $1 / 2(\mathrm{ERK} 1 / 2)$ and myosin light chain kinase (MLCK) (Iglesias et al., 2020). Urolithin A (UroA) can be detected at relatively high amounts in the colon. Its antiinflammatory activities were reported from a rat model of colitis (Larrosa et al., 2010) and a colon fibroblast model (Giménez-Bastida et al., 2012).

Intestinal inflammation causes intestinal barrier dysfunction [(reviewed in Hering et al., (2012)). The intestinal barrier integrity plays a central role for gut health by preventing an abandoned passage of antigens, allergens, bacterial toxins or other noxious agents from the intestinal lumen into the mucosa and blood circulation. The intestinal epithelium is built up by a single row of epithelial cells, which are connected by tight junctions (TJs) at their most apical point. Dependent on the physiological condition, the epithelial TJs regulates the paracellular passive passage of water and nutrients. This is achieved by the specific interplay of different TJ proteins, including the large family of claudins, TJ-associated MARVEL proteins (Mineta et al., 2011), such as tricellulin, and junctional adhesion molecules (Raleigh et al., 2010). These transmembrane proteins are connected to intracellular scaffold proteins (e.g. zonula occludens proteins, ZO-1-3) and form a meshwork of numerous horizontally oriented strands surrounding the epithelial cells. Changes to this defined composition can result in altered barrier function. Pro-inflammatory cytokines, as e.g. tumor necrosis factor a (TNFa) are well-known to cause barrier dysfunction by inducing epithelial apoptosis and by affecting TJ architecture, including claudin protein expression and delocalization (Hering and Schulzke, 2009).
So far, little is known about the impact of punicalagin, ellagic acid or urolithin A on intestinal barrier function. Objective of the present study was to elucidate their putative protective and barrier strengthening properties on epithelial TJ integrity per se or in state of inflammation. As their bioavailability differs along the gastrointestinal tract (Espín et al., 2013), we hypothesized that these bioactive compounds might act distinctly on barrier function in ileum or colon. Therefore, we investigated two different intestinal cell lines, ileum-like Caco-2 cells and HT29/B6 colon cells.

\section{MATERIALS AND METHODS}

\section{Cell Culture and Dosage Information}

Caco-2 cells are epithelial cells derived from a colorectal adenocarcinoma (ATCC ${ }^{\circledR} \mathrm{HTB}-37^{\mathrm{TM}}$ ). However, under specific culturing conditions Caco-2 cells differentiate and polarize such that they functionally and morphologically resemble the phenotype of distal ileum enterocytes. They are characterized by absorptive capabilities and active transport pathways, possess enzymatic activities and an apical brush border. When cultured on filter supports Caco-2 cells grow as polarized monolayers with epithelial TJs (Hidalgo et al., 1989).

In the present study Caco-2 cells were cultured using Minimum Essential Medium Eagle AqmediaTM containing $15 \%$ bovine serum and $1 \%$ penicillin/streptomycin (all SigmaAldrich, Schnelldorf, Germany). Within two weeks after seeding on permeable Millicell PCF filters $\left(0.6 \mathrm{~cm}^{2}\right.$ effective area; $0.4 \mu \mathrm{m}$ pores, Millicell PCF, Millipore, Schwalbach, Germany), they grew to confluence and transepithelial resistance (TER) was usually ranging between 280 and $450 \Omega \cdot \mathrm{cm}^{2}$. Fifteen days old monolayers were challenged with different doses of punicalagin $(10,25,50$, 150 and $250 \mu \mathrm{M}$; Sigma Aldrich), ellagic acid hydrate (50, 100, 150, 200 and $300 \mu \mathrm{M}$; Sigma-Aldrich) or urolithin A (25, 50, 100, 150 and $250 \mu \mathrm{M}$; Sigma-Aldrich). Substances were dissolved in dimethyl sulfoxide (DMSO). To avoid osmotic effects the monolayers were usually challenged from the apical and basolateral side. Control monolayers were treated with equal amounts of DMSO. Three aspects considered the optimal doses. 1) TER effect, 2) reproducibility (demonstrated by SEM), and 3) the amount of dimethyl sulfoxide (DMSO). Due to the toxicity of DMSO, concentrations above $1 \%$ DMSO were not considered for optimal doses.

Myosin light chain kinase inhibitor PIK $(150 \mu \mathrm{M})$ was preincubated on Caco-2 monolayers $2 \mathrm{~h}$ before challenging with EA. Phosphorylation events were studied under serum free conditions.

The colon carcinoma cell line HT-29/B6 is a subclone of the human colon carcinoma cell line HT-29 (Kreusel et al., 1991) and was cultured on permeable filter supports $\left(0.6 \mathrm{~cm}^{2}\right.$ effective area; $3.0 \mu \mathrm{m}$ pores, Millicell PCF, Millipore) using RPMI medium (Sigma-Aldrich) containing $10 \%$ bovine serum and $1 \%$ penicillin/streptomycin. Monolayers grew confluent within one week, giving a TER of at least $350 \Omega \cdot \mathrm{cm}^{2}$ and were pre-incubated with $10 \mu \mathrm{M}$ punicalagin, $150 \mu \mathrm{M}$ ellagic acid and $150 \mu \mathrm{M}$ or $250 \mu \mathrm{M}$ urolithin A from both sides. Two hours later $500 \mathrm{U} / \mathrm{ml}$ 
TNFa (Pepro Tech, Hamburg, Germany) were added to the basal compartment.

Changes in barrier integrity were assessed by measuring transepithelial resistance (TER) with a pair of chopstick electrodes at $37 \mathrm{C}$ as described before (Heller et al., 2005).

\section{Permeability Measurements}

For permeability measurements, monolayers were mounted into Ussing chambers. The standard bathing solution contained: $140 \mathrm{mM} \mathrm{Na}{ }^{+}, 123.8 \mathrm{mM} \mathrm{Cl}^{-}, 5.4 \mathrm{mM} \mathrm{K} \mathrm{K}^{+}, 1.2 \mathrm{mM} \mathrm{Ca}^{2+}$, $1.2 \mathrm{mM} \mathrm{Mg}^{2+}, 2.4 \mathrm{mM} \mathrm{HPO}_{4}{ }^{2-}, 0.6 \mathrm{mM} \mathrm{H} \mathrm{H}_{2} \mathrm{PO}_{4}{ }^{-}, 21 \mathrm{mM}$ $\mathrm{HCO}_{3}{ }^{-}$and $10 \mathrm{mM} \mathrm{D}(+)$-glucose. Flux measurements were performed under voltage-clamp conditions with $0.1 \mathrm{mM}$ fluorescein (332 Da, Sigma-Aldrich, Schnelldorf, Germany), which was added to the apical side of the monolayer. Samples were collected from the basolateral side at defined time points. Fluorescence was measured in a spectrofluorimeter (Infinite M200, Tecan, Männedorf, Austria) at $525 \mathrm{~nm}$. Fluorescein permeability $p\left(\mathrm{~cm} \cdot \mathrm{s}^{-1}\right)$ was calculated from the ratio of flux $\mathrm{J}$ $\left(\mathrm{mol} \cdot \mathrm{h}^{-1} \cdot \mathrm{cm}^{-2}\right)$ over concentration $\Delta \mathrm{c}(\mathrm{mol} / \mathrm{L}): p=\mathrm{J} / \Delta \mathrm{c}$.

For the measurement of $\mathrm{Na}^{+}$and $\mathrm{Cl}^{-}$permeability, voltage and TER were monitored while reducing $\mathrm{NaCl}$ concentration in one hemichamber. This was done by switching to a solution containing a reduced concentration of $\mathrm{NaCl}$ and mannitol for balancing osmolality. All other components were equivalent to standard solution. $\mathrm{NaCl}$ permeability was determined from dilution potentials and the Goldmann-Hodgkin-Katz equation as previously reported (Amasheh et al., 2002; Günzel et al., 2009; Yu et al., 2009).

\section{Western Blot Analyses}

For expression analyses, proteins were extracted using ice-cold lysis buffer, including $10 \mathrm{mM}$ Tris, $\mathrm{pH}$ 7.5, $150 \mathrm{mM} \mathrm{NaCl}, 0.5 \%$ Triton X-100, 0.1\% SDS and complete protease inhibitor mixture (Roche, Basel, Switzerland). For phosphorylation studies, the lysis buffer contained $20 \mathrm{mM}$ Tris ( $\mathrm{pH} 7.5$ ), $150 \mathrm{mM} \mathrm{NaCl}, 1 \mathrm{mM}$ EDTA, $1 \mathrm{mM}$ EGTA, $1 \%$ Triton X-100, $2.5 \mathrm{mM} \mathrm{Na} \mathrm{H}_{2} \mathrm{P}_{2} \mathrm{O}_{7}$, $1 \mathrm{mM} \beta$-glycerolphosphate, $1 \mathrm{mM} \mathrm{Na}_{3} \mathrm{VO}_{4}, 1 \mathrm{mg} \mathrm{ml}^{-1}$ leupeptin, $1 \mathrm{mM}$ PMSF and complete protease inhibitor. For analysis of caspase- 3 cleavage, cell lysis was performed as described recently (Hering et al., 2017).

Protein extracts (15-40 mg) were separated by SDS-gel electrophoresis and blotted on PVDF membrane. Antibodies used for immunodetection: anti-claudin-1 to -4 , claudin-7 and -15 (1:1000, Thermo Fisher Scientific, Bremen, Germany), $\beta$-actin (1:5000, Sigma-Aldrich), anti-phospho-myosin light chain 2, anti-myosin light chain 2 (Cell Signaling Technology, Danvers, MA, USA), and anti-caspase 3 (1:1000; Cell Signaling Technology). Chemiluminescent imaging of bound antibodies was performed with peroxidase-conjugated goat anti-rabbit IgG or goat anti-mouse IgG antibodies, chemiluminescence substrate Lumi-LightPLUS (Roche) and the FX7detection system (Vilber Lourmat, Eberhardzell, Germany). Densitometry was carried out with Image Studio Light (LI-COR Biosciences; NE, United States) and values were normalized to $\beta$-actin that served as internal loading control.

\section{Immunofluorescence Staining and Confocal Laser Scanning Microscopy}

Monolayers were rinsed with PBS and fixed with $4 \%$ of paraformaldehyde for $10 \mathrm{~min}$ at room temperature. After permeabilization with $0.5 \%$ Triton X-100 monolayers were blocked with $5 \%$ goat serum and $1 \%$ bovine serum albumin. Immunostaining was carried out with primary antibodies for anti-ZO-1 (1:100), anti-claudin-1, $-4,-7$ and -15 (all 1:100; Thermo Fisher Scientific) at $4^{\circ} \mathrm{C}$ over night. Counterstaining was performed using Alexa Fluor 488 goat anti-mouse and Alexa Fluor 594 goat anti-rabbit IgG (1:1000; Thermo Fisher Scientific) as described before (Luettig et al., 2016). Nuclei were stained with 4',6-Diamidin-2-phenylindol (DAPI) (1:5000). Intensity and localization of claudins was analyzed by confocal laser scanning microscopy (LSM 780, Zeiss, Jena, Germany).

\section{Freeze Fracture Electron Microscopy}

Freeze fracture electron microscopy analysis was performed and quantified as described elsewhere in detail (Krug et al., 2009).

\section{Statistical Analyses}

Statistical analysis was done using Student's t-test and Bonferroni-Holm adjustment in case of multiple comparison. All data are expressed as mean \pm SEM. $p<0.05$ was considered significant $\left({ }^{*} p<0.05 ;{ }^{* *} p<0.01 ;{ }^{* *} p<0.001\right.$ or ${ }^{*} p<0.05$; \#\# $p<0.01$; \#\# $p<0.001)$.

\section{RESULTS}

\section{Stabilizing Effect of Punicalagin, Urolithin A and Ellagic Acid on Barrier Properties of Caco-2 Intestinal Cells}

Punicalagin increased TER slightly from initial values in Caco-2 monolayers within 24h (Figure 1A; $p<0.05, p<0.001$ vs. control). This TER increase could not be enhanced dosedependently (Figure 1A; $p<0.05$ Puni 50 vs. $150 \mu \mathrm{M}$ ). In contrast, the TER increase induced by urolithin A was more pronounced (Figure 1B). While $25 \mu \mathrm{M}$ was not effective, doses up to $100 \mu \mathrm{M}$ UroA increased TER significantly from control (Figure 1B; $p<0.001$ vs. control). Higher doses than $100 \mu \mathrm{M}$ of urolithin A had no further increasing effects (Figure 1B). Ellagic acid caused the strongest TER increase within 24 h. $50 \mu \mathrm{M}$ was as effective as $200 \mu \mathrm{M}$ EA compared to control (Figure 1C; $p<0.001$ and $p<0.05$ vs. control). Comparing the most effective dose of each compound in one experiment proved ellagic acid $(150 \mu \mathrm{M})$ to induce the strongest TER increase in Caco-2 monolayers, followed by urolithin $\mathrm{A}(250 \mu \mathrm{M})$ and punicalagin $(10 \mu \mathrm{M})$ (Figure 1D; $p<0.001$ vs. control and $p<0.001$ vs. EA). The TER increase induced by $150 \mu \mathrm{M}$ ellagic acid was paralleled by a permeability decrease of the $332 \mathrm{Da}$ marker molecule fluorescein in Caco-2 monolayers (Figure 2A; $p<0.001$ vs. control). Neither $250 \mu \mathrm{M}$ urolithin A nor $10 \mu \mathrm{M}$ punicalagin reduced fluorescein permeability (Figure 2A). Measurements 

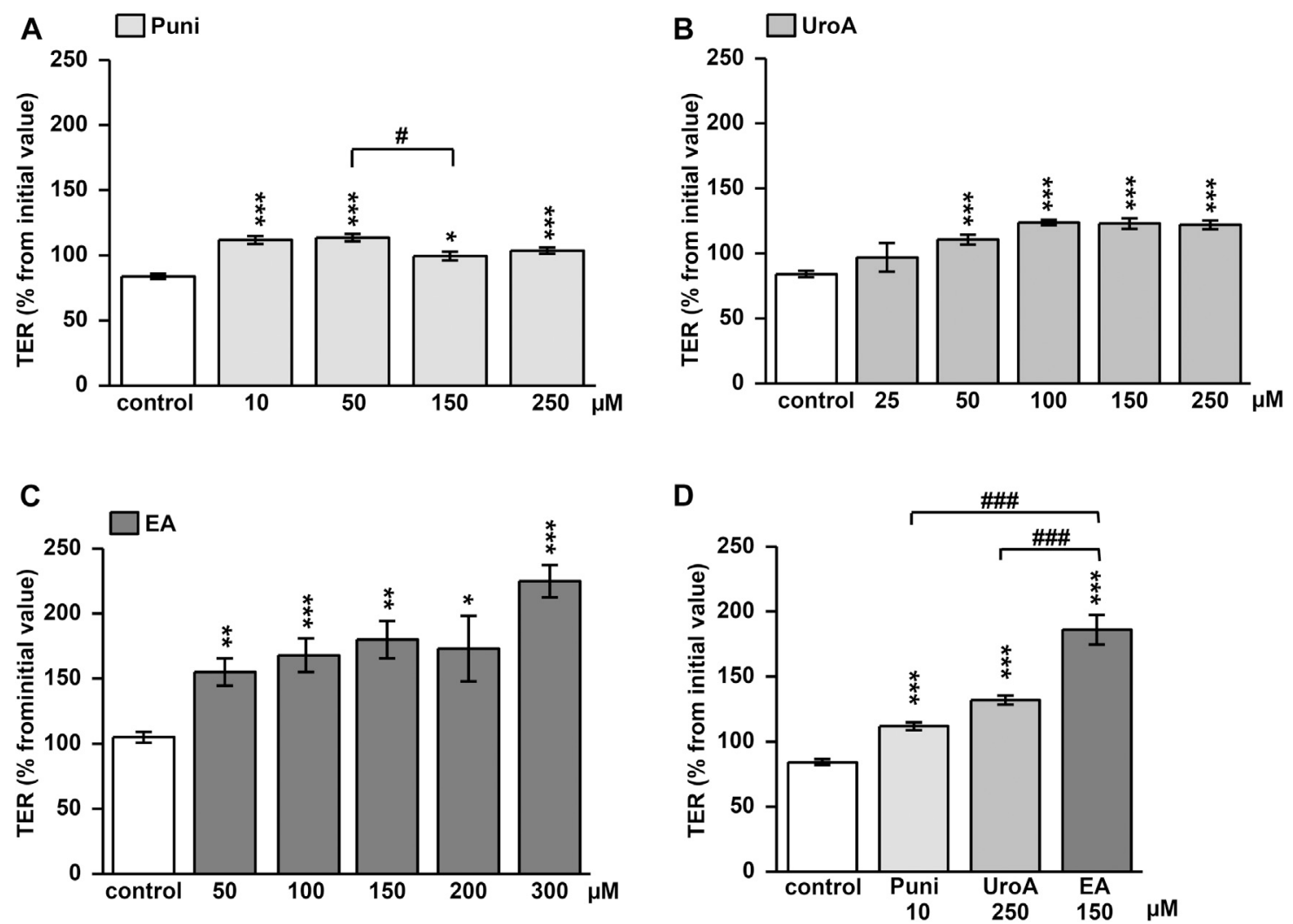

FIGURE 1 | Impact of punicalagin, ellagic acid and urolithin A on transepithelial resistance (TER) in Caco-2 monolayers. Changes in TER from initial values were measured at $24 \mathrm{~h}$ after challenging monolayers with different doses of (A) punicallagin (Puni) $\left(p^{*}<0.05,{ }^{* \star *} p<0.001\right.$ vs. control; ${ }^{\#} p<0.0550$ vs. $\left.150 \mu \mathrm{M} ; n=3-12\right)$ (B) urolithin $\mathrm{A}$ (UroA) ${ }^{* \star *} p<0.001$ vs. control; $\left.n=3-12\right)$ or $(\mathbf{C})$ ellagic acid (EA) $\left(p^{*}<0.05,{ }^{* *} p<0.01,{ }^{* \star *} p<0.001\right.$ vs. control; $\left.n=6-15\right)$ (D) Comparison of the most effective dose of each compound on TER at $24 \mathrm{~h}$ after challenging $\left({ }^{\star \star *} p<0.001\right.$ vs. control; ${ }^{\# \#} p<0.001$ vs. ellagic acid; $\left.n=6-9\right)$.
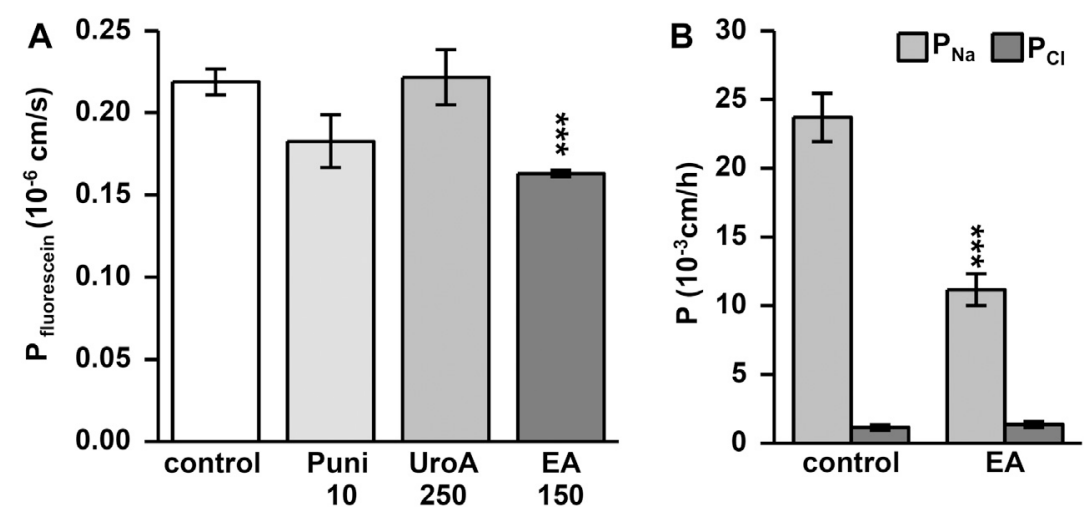

FIGURE 2 |Ellagic acid decreases permeability to fluorescein and sodium in Caco-2 monolayers. Permeability measurements were performed in Ussing chambers at $24 \mathrm{~h}$ after challenging. Ellagic acid (EA) reduces permeability to the (A) 332 Da marker molecule fluorescein $\left(^{\star \star \star} p<0.001\right.$ vs. control; $\left.n=7-9\right)$ and (B) to sodium ions ${ }^{* \star *} p<0.001$ vs. control; $\left.n=8\right)$ compared to untreated controls.

of dilution potentials for sodium and chloride showed that ellagic acid restricted sodium permeability, but not chloride permeability in Caco-2 monolayers (Figure 2B; $p<0.001$ vs. control). The permeability ratios of sodium and chloride
$\left(\mathrm{P}_{\mathrm{Na}} / \mathrm{P}_{\mathrm{Cl}}\right)$ were reduced 3 -fold from $28 \pm 7$ in control to $9 \pm 1$ in monolayers challenged with ellagic acid ( $p<0.05$ vs. control).

To analyze the barrier effect of ellagic acid in more detail, TJ protein expression was examined after $24 \mathrm{~h}$ of challenging 
A control EA

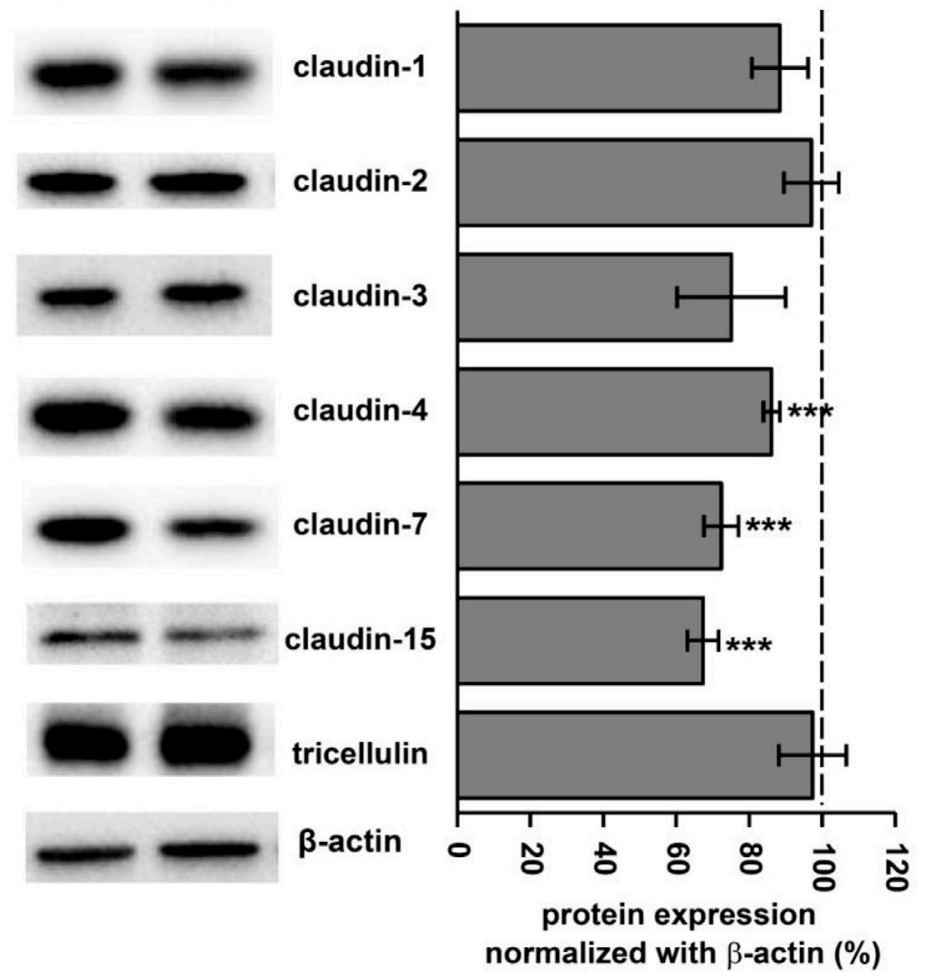

B

claudin-7
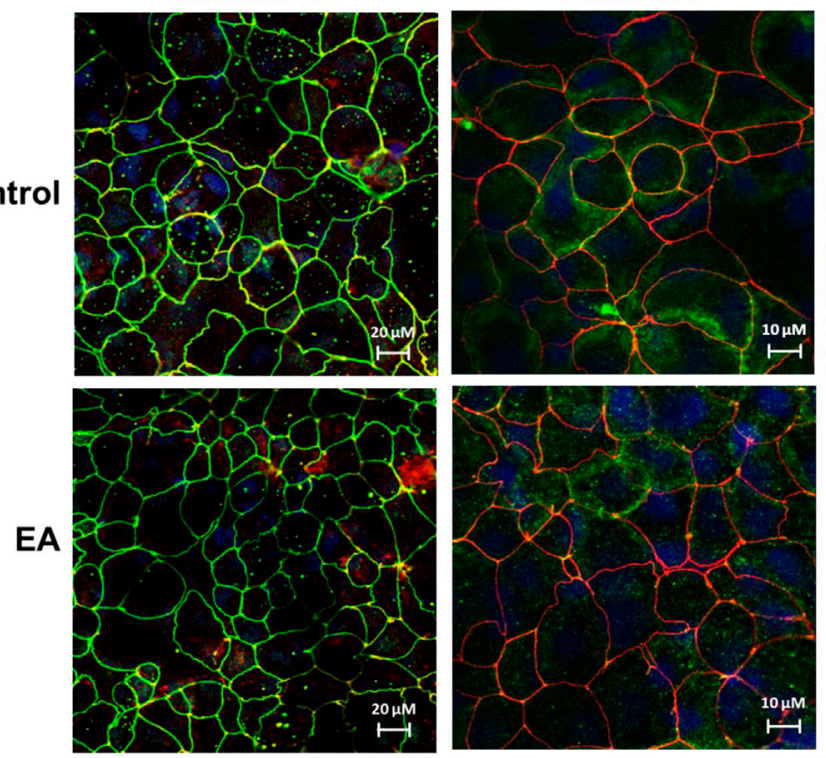

claudin

claudin-4

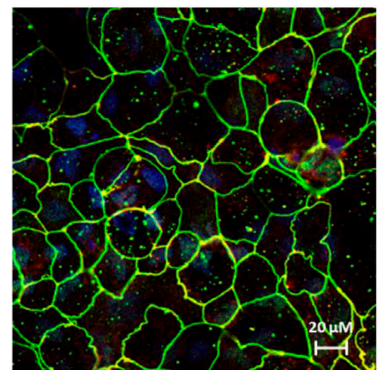

ZO-1 claudin-15
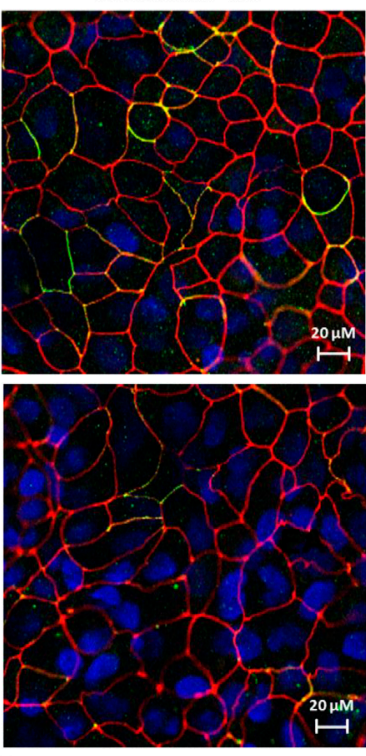

DAPI
C
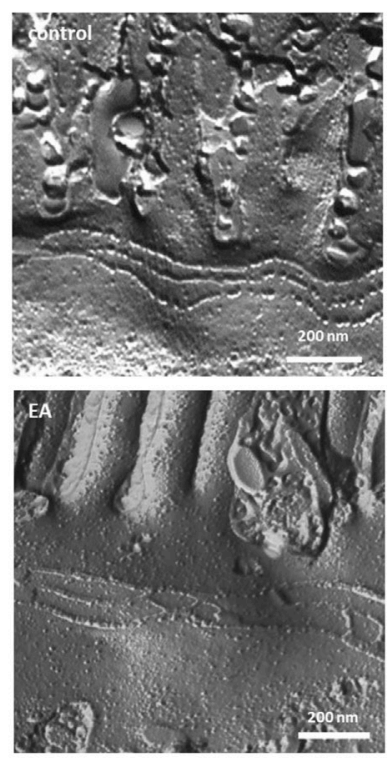

FIGURE 3 | Ellagic acid reduces protein expression of TJ proteins claudin-4, - 7 and -15 in Caco-2 monolayers (A) Representative Western blots and densitometry show reduced expression of claudin-4, -7 and -15 in ellagic acid (EA)-challenged monolayers ( $p^{\star \star \star}<0.001$ vs. control; $\left.n=7-10\right)$ (B) Representative micrographs of immunofluorescence staining show claudin-4, claudin-7 and claudin-15 in control and elllagic acid-challenged monolayers (green). ZO-1 served as TJ marker (red), nuclei were DAPI stained (blue) $(n=3$ each) (C) Representative freeze-fracture EM micrographs of control and ellagic acid-challenged monolayers show no differences in TJ ultra-structure. 

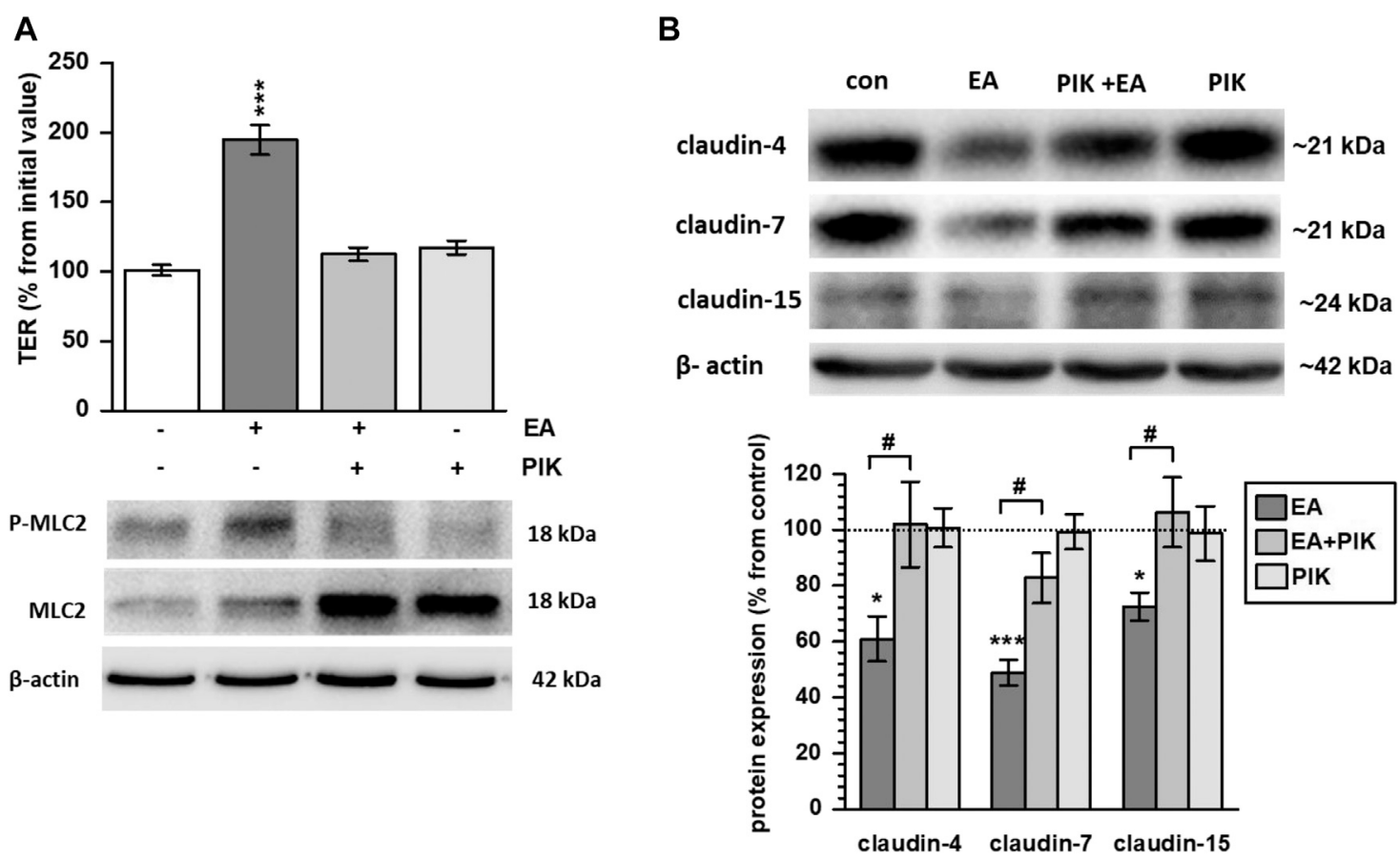

FIGURE 4 | Ellagic acid strengthens barrier function via MLC2 signaling in Caco-2 monolayers (A) Pre-incubation with the MLCK specific inhibitor PIK impeded ellagic acid (EA)-stimulated MLC2 phosphorylation and TER increase in Caco-2 monolayers at $24 \mathrm{~h}$ after challenge $\left(p^{\star \star \star}<0.001\right.$ vs. control; $\left.n=9\right)$., (B) Representative Western blots and quantification show that PIK inhibition blocked ellagic acid-stimulated down regulation of claudin-4, -7 and -15 in parallel $\left({ }^{\star} p<0.05 ;{ }^{\star \star \star} p<0.001\right.$ vs. control; ${ }^{\#} p<0.05$ ellagic acid vs. ellagic acid + PIK; $\left.n=6-9\right)$.

monolayers with $150 \mu \mathrm{M}$ ellagic acid. This reduced the protein level of claudin- $4,-7$ and -15 (Figure $3 \mathrm{~A} ; p<0.0001$ vs. control), but did not affect claudin-1, -2 or -3 and tricellulin (Figure $3 \mathbf{A}$ ). Claudin-5 and -8 were not expressed in our Caco- 2 cells. Expression down regulation of claudin-4, -7 and -15 obtained from Western blot analyses were confirmed by confocal laser scanning microscopy of immunostained Caco-2 monolayers. The intensity of claudin-4, claudin-7 and claudin-15 signals was reduced in monolayers challenged with $150 \mu \mathrm{M}$ ellagic acid (Figure 3B). Especially claudin-7 and -15 were only present in single cells of the Caco-2 control monolayers. Ellagic acid treatment reduced the number and frequency of these claudin7 or claudin-15 positive cells (Figure 3B). Overall TJ ultrastructure was not influenced by ellagic acid. Morphometric analyses of freeze fracture electron micrographs revealed no alterations in TJ ultra-structure (Figure $3 \mathrm{C}$ ). TJ strand number $(3.4 \pm 0.2$ vs. $3.2 \pm 0.1$ in control), density $(23 \pm 3$ vs. $22 \pm 2$ in control) and type, meshwork depth (147 \pm 17 vs. $143 \pm 9$ in control), and number of strand breaks did not differ from control in ellagic acid-challenged Caco-2 monolayers.

Signaling was studied by assessing different kinase inhibitors. Although ellagic acid induced phosphorylation of p38 and STAT3, specific phosphorylation inhibition of these kinases could not impede the ellagic acid-stimulated TER increase (data not shown). In contrast, inhibition of MLCK by PIK prevented Myosin Light Chain 2 (MLC2) phosphorylation and blocked the ellagic acid-induced TER increase
(Figure 4A; $p<0.001$ vs. control). In parallel, PIK impeded the ellagic acid-depended expression down-regulation of claudin-4, -7 and -15 (Figure 4B; $p<0.05$ PIK + ellagic acid vs. ellagic acid alone). In PIK + ellagic acid co-treated monolayers, protein levels did not differ from control (Figure 4B).

\section{Protective Effect of Urolithin A on TNFa-Induced Barrier Loss in HT-29/B6 Cells}

HT-29/B6 monolayers were challenged with the proinflammatory cytokine TNFa that caused a TER drop of about $40 \%$ within $24 \mathrm{~h}$ (Figure 5A; $p<0.001$ vs. control). Pretreatment with $150 \mu \mathrm{M}$ or $250 \mu \mathrm{M}$ urolithin A partially reversed this TNFainduced decrease ( $p<0.001$ vs. TNFa), while both urolithin A doses were comparably effective (Figure 5A). In contrast, $10 \mu \mathrm{M}$ punicalagin or $150 \mu \mathrm{M}$ ellagic acid did not inhibit the TNFainduced TER decrease (Figure $\mathbf{5 A}$ ). TNFa is well known to upregulate expression of claudin-1 and -2 in HT-29/B6 cells. Within the present study, pre-incubation with $150 \mu \mathrm{M}$ urolithin A prevented the TNFa-stimulated up-regulation of both claudins. Western blotting showed an increase of about $40 \%$ in claudin- 1 expression by TNFa (Figure 5B; $p<0.001$ vs. control), while in urolithin A co-treated monolayers expression remained at the control level (Figure 5B). Claudin-2 protein level was increased by $\mathrm{TNF} \alpha$ about $50 \%$ from control values (Figure 5B; $p<0.01$ vs. control). Pre-treatment with urolithin A reduced claudin-2 


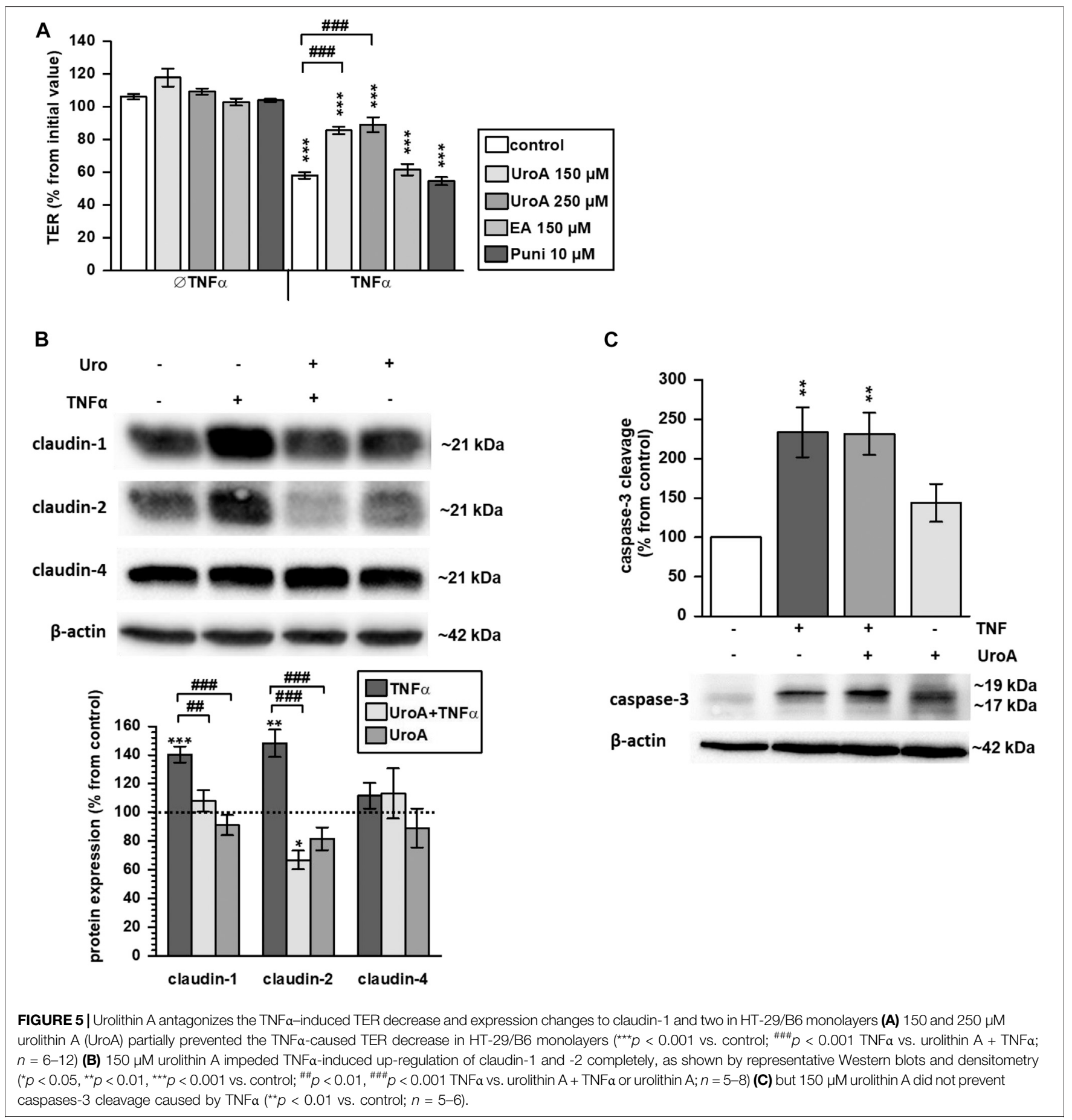

expression to $67 \%$ from control (Figure $5 \mathrm{~B} ; p<0.05$ vs. control). Claudin-4 expression was not affected by TNFa or urolithin A (Figure 5B).

Because TNFa causes epithelial apoptosis in HT-29/B6 cells, effects of urolithin A on caspase-3 cleavage were examined by Western blotting. As expected, TNFa enhanced caspase-3 cleavage compared to untreated controls (Figure 5C; $p<0.01$ vs. control). Urolithin A did not reduce TNFa-induced caspase-3 cleavage, but seemed to stimulate it. However, this did not reach statistical significance (Figure 5C). Beside expression regulation, TNFa caused claudin-1 redistribution out off the TJ into subapical compartments compared to untreated controls (Figures 6A,B, indicated by white arrows in Figure 6B). Representative micrographs of immunofluorescence staining showed increased merging of claudin-1 with the TJ marker protein ZO-1 in monolayers that were co-challenged with TNFa and urolithin A (indicated by white arrows in Figure 6C). In urolithin A-challenged monolayers, claudin-1 
A
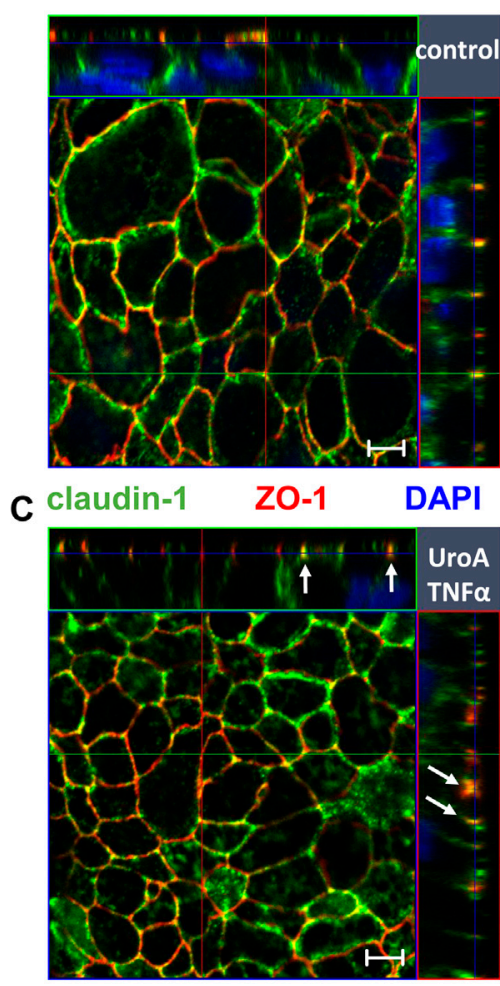
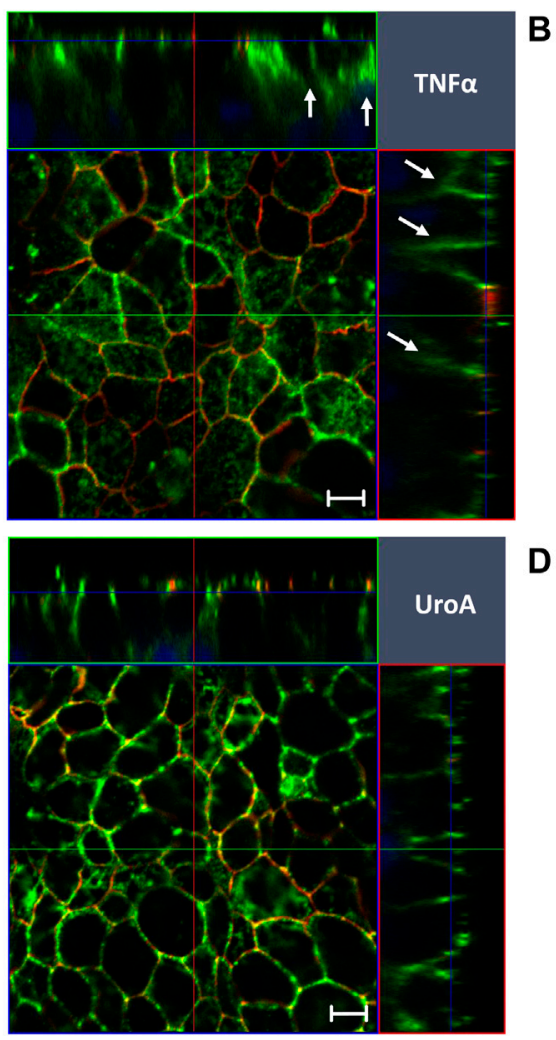

FIGURE 6 | Effect of Urolithin A on TNF $\alpha$-caused claudin-1 delocalization in HT-29/B6 monolayers. Localization of claudin-1 was studied by confocal laser scanning microscopy in (A) control (B) TNFa (C) urolithin A (UroA) +TNFa and (D) urolithin A-challenged monolayers. Merging of claudin-1 (green) with the TJ marker protein ZO-1 (red) was assessed by z-stack imaging, nuclei are DAPI stained (blue). TNFa caused delocalization of claudin-1 (indicated by white arrows in (B)), while parallel Urolithin A treatment enhanced claudin-1-ZO-1 merging (indicated by white arrows in (C)). Bars indicate $5 \mu$ m.

intensity and localization appeared not different from control (Figure 6D).

\section{DISCUSSION}

Epithelial barrier function is a key feature of intestinal health. The present study examined the molecular mechanisms behind the health claims of the bioactive polyphenol punicalagin and its metabolites ellagic acid and urolithin A on epithelial barrier function in vitro. Our data revealed that especially ellagic acid and urolithin A affect barrier function in different ways in our two cell models. Ellagic acid strengthened barrier function per se by reducing the expression of pore-forming claudin-4, -7 and -15 via MLC2 signaling in ileum-like Caco-2 cells. Urolithin A impeded the pro-inflammatory dysregulation and/or redistribution of claudin-2 and -1 in colonic HT-29/B6 cells. Punicalagin alone had only little or no effects on barrier function in the two cell models.

The constitution of the epithelial TJ, particularly its composition of pore-forming and sealing TJ proteins, is crucial for intestinal barrier function. Barrier integrity can be estimated by measuring TER and paracellular permeability of differently sized molecules or ions. Especially in Caco-2 cells, ellagic acid turned out to strengthen epithelial barrier function per se, displayed by a strong increase in TER, reduction in fluorescein and sodium permeability and down regulation of claudin-4, -7 and -15 expression.

Tight junction ultra-structure was not changed as demonstrated by freeze fracture electron microscopy. Subsequently, barrier effects are not due to changes in overall protein content but to TJ protein composition. Especially, the downregulation of channel forming claudins seems rational. Claudin-15 is predominantly expressed in the small intestine (Inai et al., 2005; Fujita et al., 2006; Holmes et al., 2006) and forms a paracellular cation- (Colegio et al., 2002; Van Itallie et al., 2003; Samanta et al., 2018) and water-selective channel (Rosenthal et al., 2020). Overexpression of claudin-15 in Caco-2 cells caused a decrease in TER (Takehara et al., 2009). The physiological function of claudin- 4 and -7 is less clear as it is not consistent and seems to depend on the interactions with other $\mathrm{TJ}$ proteins and differs in different cell types and conditions (Günzel and Fromm, 2012). Two studies on flavonoids in Caco-2 cells reported quercetin (Amasheh et al., 2008) or kaempferol (Suzuki et al., 2011) to enhance barrier function by up-regulating claudin-4 expression. In contrast, in a cell culture study on kidney cells, claudin- 4 was suggested to act as a chloride channel. Knock down of claudin- 4 resulted here in a decrease in $\mathrm{P}_{\mathrm{Na}} / \mathrm{P}_{\mathrm{Cl}}$, which 
was paralleled by an TER increase (Hou et al., 2010). In an epithelial co-culture model of Caco and HT29-MTX cells, a decrease in TER induced by oxidative stress was associated with an increase in claudin-7 (Bianchi et al., 2019). In kidney cells, claudin-7 overexpression was associated with a decrease in $\mathrm{P}_{\mathrm{Cl}}$ and an increase in $\mathrm{P}_{\mathrm{Na}}$ (Alexandre et al., 2005). Taken together, it seems rational that especially the reduction of claudin-15 and claudin-7 are responsible for the decrease in sodium permeability and the TER increase in ellagic acidchallenged Caco-2 monolayers.

Ellagic acid enhanced the phosphorylation of STAT3, p38 and MLC2 in Caco-2 cells. However, only inhibition of MLC2 phosphorylation by the MLCK-inhibitor PIK impeded the ellagic acid-stimulated TER increase, suggesting a central role of MLC2 in ellagic acid-dependent barrier regulation. Phosphorylation of the MLC2 by MLCK is linked to actomyosin contraction and TJ regulation (Turner et al., 1999; Zolotarevsky et al., 2002; Shen et al., 2006). We showed that phosphorylation inhibition of MLC2 impeded the ellagic acid-induced TER increase and down-regulation of claudin-4, -7 and -15 . So far, the role of non-muscle MLCK and MLC2 for barrier function was predominantly discussed with respect to TNFa-caused barrier loss (Zolotarevsky et al., 2002; Wang et al., 2006; Ye and Ma, 2008). Iglesias et al., recently showed ellagic acid to inhibit TNFa-stimulated MLC2 phosphorylation (Iglesias et al., 2020). In contrast, our data show the activation of MLCK/MLC2 in a none-inflammatory state and suggest that MLC2-triggered TJ regulation is not necessarily related to barrier loss as it seems to depend on the specific type of TJ protein affected.

Within the present study, the impact of punicalagin, ellagic acid and urolithin $A$ on inflammation-induced barrier dysfunction was examined in the HT-29/B6 cell culture model, which is a very well-studied inflammation model and more sensitive to TNFa than Caco-2 cells. TNFa is known to upregulate the expression of sealing claudin-1 and channel forming claudin-2, at which claudin-1 is additionally redistributed from the TJ in HT-29/B6. Together with enhanced epithelial apoptosis, these TJ changes are reported to critically contribute to the TNFacaused barrier loss (Gitter et al., 2000; Mankertz et al., 2009; Amasheh et al., 2010). In contrast to urolithin A, neither punicalagin nor ellagic acid were effective to inhibit the TNFa-induced TER drop in HT-29/B6. Moreover, urolithin A impeded up-regulation of caudin- 1 and -2 and seemed at least partially to prevent redistribution of claudin-1. These effects of urolithin A are very similar to effects we observed in a former study on the ginger-derived pungent component 6-shogaol that also impeded the TNFa-induced up-regulation of claudin- 2 and disassembly of claudin-1 (Luettig et al., 2016). Furthermore, TNFa enhances epithelial apoptosis contributing to epithelial leakiness (Gitter et al., 2000). Urolithin A did not inhibit TNFacaused epithelial apoptosis in HT-29/B6, but even seemed to stimulate it slightly. This is in concordance with other studies that showed ellagitannins from pomegranate and urolithin metabolites to inhibit proliferation and to induce apoptosis in HT-29 cells (Kasimsetty et al., 2010).
These very distinct effects of punicalagin, ellagic acid and urolithin A on intestinal barrier function in the two cell models seem rational, because the bioavailability of these compounds differs along the intestine. Punicalagin was reported to be hydrolyzed already during the stomach passage where it yields ellagic acid. Punicalagin itself probably does not reach the intestine in high amounts, while ellagic acid might predominantly interact with the enterocytes of the ileum. As reported from clinical trials, ellagic acid was not detected in high amounts in the colonic mucosa (Nuñez-Sánchez et al., 2014). In contrast, an increasing gradient of urolithins from the jejunum to the distal colon was described in an animal study (Espín et al., 2007). So far, only very few studies addressed the question, how much punicalagin has to be ingested to reach effective intestinal concentrations of ellagic acid or urolithin A. In the present study, the effects seemed to depend on the optimal dosage, which was figured out for each compound. González-Sarrías et al. simulated gastrointestinal digestion of pomegranate extracts yielding around $500 \mu \mathrm{M}$ ellagic acid, while plasma concentrations remained low at $100 \mathrm{nM}$ (Nuñez-Sánchez et al., 2014). Subsequently, there are more studies needed to elucidate dosage, intestinal side of conversion, and bioavailability of these components in vivo.

\section{CONCLUSION}

Our study reveals that the punicalagin metabolites ellagic acid and urolithin A have a protective impact on barrier function in vitro. These findings support the hypothesis that therapeutically application might act preventive by strengthening and protecting the epithelial barrier in case of diarrhea or inflammation. Moreover, the characterization of these compounds might be of interest for the development of multimodal functional food in the future.

\section{DATA AVAILABILITY STATEMENT}

The raw data supporting the conclusions of this article will be made available by the authors, without undue reservation.

\section{AUTHOR CONTRIBUTIONS}

$\mathrm{NH}, \mathrm{RR}$ and JS conceived and designed research project; JL, NH, $\mathrm{RR}$ and $\mathrm{BJ}$ conducted research; $\mathrm{NH}$ and RR analyzed data; $\mathrm{NH}$ performed statistical analysis; NH and RR wrote the paper; and JS and RR had primary responsibility for final content. All authors read and approved the final manuscript.

\section{ACKNOWLEDGMENTS}

This study was supported by grants from Deutsche Forschungsgemeinschaft (DFG) Schu 559/11. 


\section{REFERENCES}

Alexandre, M. D., Lu, Q., and Chen, Y. H. (2005). Overexpression of claudin-7 decreases the paracellular $\mathrm{Cl}$ - conductance and increases the paracellular $\mathrm{Na}+$ conductance in LLC-PK1 cells. J. Cel. Sci 118 (12), 2683-2693. doi:10.1242/jcs. 02406

Amasheh, M., Fromm, A., Krug, S. M., Amasheh, S., Andres, S., Zeitz, M., et al. (2010). TNFalpha-induced and berberine-antagonized tight junction barrier impairment via tyrosine kinase, Akt and NFkappaB signaling. J. Cel. Sci 123 (23), 4145-4155. doi:10.1242/jcs.070896

Amasheh, M., Schlichter, S., Amasheh, S., Mankertz, J., Zeitz, M., Fromm, M., et al. (2008). Quercetin enhances epithelial barrier function and increases claudin-4 expression in Caco-2 cells. J. Nutr. 138 (6), 1067-1073. doi:10.1093/jn/138.6. 1067

Amasheh, S., Meiri, N., Gitter, A. H., Schöneberg, T., Mankertz, J., Schulzke, J. D., et al. (2002). Claudin-2 expression induces cation-selective channels in tight junctions of epithelial cells. J. Cel. Sci. 115 (24), 4969-4976. doi:10.1242/jcs. 00165

Bianchi, M. G., Chiu, M., Taurino, G., Brighenti, F., Del Rio, D., Mena, P., et al. (2019). Catechin and procyanidin B2 modulate the expression of tight junction proteins but do not protect from inflammation-induced changes in permeability in human intestinal cell monolayers. Nutrients 11 (10), 2271. doi:10.3390/nu11102271

Cerdá, B., Periago, P., Espín, J. C., and Tomás-Barberán, F. A. (2005). Identification of urolithin a as a metabolite produced by human colon microflora from ellagic acid and related compounds. J. Agric. Food Chem. 53 (14), 5571-5576. doi:10. $1021 / \mathrm{jf} 050384 \mathrm{i}$

Colegio, O. R., Van Itallie, C. M., McCrea, H. J., Rahner, C., and Anderson, J. M. (2002). Claudins create charge-selective channels in the paracellular pathway between epithelial cells. Am. J. Physiol, Cel. Physiol. 283 (1), C142-C147. doi:10. 1152/ajpcell.00038.2002

Das, A. K., Mandal, S. C., Banerjee, S. K., Sinha, S., Das, J., Saha, B. P., et al. (1999). Studies on antidiarrhoeal activity of Punica granatum seed extract in rats. J. Ethnopharmacol 68 (1-3), 205-208. doi:10.1016/s0378-8741(99) 00102-6

Espín, J. C., González-Barrio, R., Cerdá, B., López-Bote, C., Rey, A. I., and TomásBarberán, F. A. (2007). Iberian pig as a model to clarify obscure points in the bioavailability and metabolism of ellagitannins in humans. J. Agric. Food Chem. 55 (25), 10476-10485. doi:10.1021/jf0723864

Espín, J. C., Larrosa, M., García-Conesa, M. T., and Tomás-Barberán, F. (2013) Biological significance of urolithins, the gut microbial ellagic Acid-derived metabolites: the evidence so far. Evid. Based Complement. Alternat Med. 2013, 270418. doi:10.1155/2013/270418

Fujita, H., Chiba, H., Yokozaki, H., Sakai, N., Sugimoto, K., Wada, T., et al. (2006). Differential expression and subcellular localization of claudin-7, -8, -12, -13, and -15 along the mouse intestine. J. Histochem. Cytochem. 54 (8), 933-944. doi:10.1369/jhc.6A6944.2006

Giménez-Bastida, J. A., González-Sarrías, A., Larrosa, M., Tomás-Barberán, F., Espín, J. C., and García-Conesa, M. T. (2012). Ellagitannin metabolites, urolithin A glucuronide and its aglycone urolithin A, ameliorate TNFa-induced inflammation and associated molecular markers in human aortic endothelial cells. Mol. Nutr. Food Res. 56 (5), 784-796. doi:10.1002/mnfr. 201100677

Gitter, A. H., Bendfeldt, K., Schulzke, J. D., and Fromm, M. (2000). Leaks in the epithelial barrier caused by spontaneous and TNF-alpha-induced single-cell apoptosis. Faseb J. 14 (12), 1749-1753. doi:10.1096/fj.990898com

Günzel, D., and Fromm, M. (2012). Claudins and other tight junction proteins. Compr. Physiol. 2 (3), 1819-1852. doi:10.1002/cphy.c110045

Günzel, D., Stuiver, M., Kausalya, P. J., Haisch, L., Krug, S. M., Rosenthal, R., et al. (2009). Claudin-10 exists in six alternatively spliced isoforms that exhibit distinct localization and function. J. Cel. Sci. 122 (10), 1507-1517. doi:10. $1242 /$ jcs. 040113

Heller, F., Florian, P., Bojarski, C., Richter, J., Christ, M., Hillenbrand, B., et al. (2005). Interleukin-13 is the key effector Th2 cytokine in ulcerative colitis that affects epithelial tight junctions, apoptosis, and cell restitution. Gastroenterology 129 (2), 550-564. doi:10.1016/j.gastro.2005.05.002
Hering, N. A., Fromm, M., and Schulzke, J. D. (2012). Determinants of colonic barrier function in inflammatory bowel disease and potential therapeutics. J. Physiol. 590 (5), 1035-1044. doi:10.1113/jphysiol.2011.224568

Hering, N. A., Luettig, J., Krug, S. M., Wiegand, S., Gross, G., van Tol, E. A., et al. (2017). Lactoferrin protects against intestinal inflammation and bacteriainduced barrier dysfunction in vitro. Ann. N. Y Acad. Sci. 1405 (1), 177-188. doi:10.1111/nyas. 13405

Hering, N. A., and Schulzke, J. D. (2009). Therapeutic options to modulate barrier defects in inflammatory bowel disease. Dig. Dis. 27 (4), 450-454. doi:10.1159/ 000233283

Hidalgo, I. J., Raub, T. J., and Borchardt, R. T. (1989). Characterization of the human colon carcinoma cell line (Caco-2) as a model system for intestinal epithelial permeability. Gastroenterology 96 (3), 736-749. doi:10.1016/00165085(89)90897-4

Holmes, J. L., Van Itallie, C. M., Rasmussen, J. E., and Anderson, J. M. (2006). Claudin profiling in the mouse during postnatal intestinal development and along the gastrointestinal tract reveals complex expression patterns. Gene Expr. Patterns 6 (6), 581-588. doi:10.1016/j.modgep.2005.12.001

Hou, J., Renigunta, A., Yang, J., and Waldegger, S. (2010). Claudin-4 forms paracellular chloride channel in the kidney and requires claudin- 8 for tight junction localization. Proc. Natl. Acad. Sci. USA 107 (42), 18010-18015. doi:10. 1073/pnas.1009399107

Iglesias, D. E., Cremonini, E., Fraga, C. G., and Oteiza, P. I. (2020) Ellagic acid protects Caco-2 cell monolayers against inflammation-induced permeabilization. Free Radic. Biol. Med. 152, 776-786. doi:10.1016/j. freeradbiomed.2020.01.022

Inai, T., Sengoku, A., Guan, X., Hirose, E., Iida, H., and Shibata, Y. (2005) Heterogeneity in expression and subcellular localization of tight junction proteins, claudin-10 and -15 , examined by RT-PCR and immunofluorescence microscopy. Arch. Histol. Cytol. 68 (5), 349-360. doi:10.1679/aohc.68.349

Kasimsetty, S. G., Bialonska, D., Reddy, M. K., Ma, G., Khan, S. I., and Ferreira, D. (2010) Colon cancer chemopreventive activities of pomegranate ellagitannins and urolithins. J. Agric. Food Chem. 58 (4), 2180-2187. doi:10.1021/jf903762h

Kreusel, K. M., Fromm, M., Schulzke, J. D., and Hegel, U. (1991) Cl- secretion in epithelial monolayers of mucus-forming human colon cells (HT-29/B6). Am. J. Physiol. 261, C574-C582. doi:10.1152/ajpcell.1991.261.4.C574

Krug, S. M., Amasheh, S., Richter, J. F., Milatz, S., Günzel, D., Westphal, J. K., et al. (2009). Tricellulin forms a barrier to macromolecules in tricellular tight junctions without affecting ion permeability. Mol. Biol. Cel. 20 (16), 3713-3724. doi:10.1091/mbc.E09-01-0080

Larrosa, M., González-Sarrías, A., Yáñez-Gascón, M. J., Selma, M. V., AzorínOrtuño, M., Toti, S., et al. (2010). Anti-inflammatory properties of a pomegranate extract and its metabolite urolithin-A in a colitis rat model and the effect of colon inflammation on phenolic metabolism. J. Nutr. Biochem. 21 (8), 717-725. doi:10.1016/j.jnutbio.2009.04.012

Longtin, R. (2003). The pomegranate: nature's power fruit? J. Natl. Cancer Inst. 95 (5), 346-348. doi:10.1093/jnci/95.5.346

Luettig, J., Rosenthal, R., Lee, I. M., Krug, S. M., and Schulzke, J. D. (2016). The ginger component 6-shogaol prevents TNF- $\alpha$-induced barrier loss via inhibition of PI3K/Akt and NF-kB signaling. Mol. Nutr. Food Res. 60 (12), 2576-2586. doi:10.1002/mnfr.201600274

Mankertz, J., Amasheh, M., Krug, S. M., Fromm, A., Amasheh, S., Hillenbrand, B., et al. (2009). TNFalpha up-regulates claudin-2 expression in epithelial HT-29/ B6 cells via phosphatidylinositol-3-kinase signaling. Cel. Tissue Res 336, 67. doi:10.1007/s00441-009-0751-8

Marín, M., María Giner, R., Ríos, J. L., and Recio, M. C. (2013). Intestinal antiinflammatory activity of ellagic acid in the acute and chronic dextrane sulfate sodium models of mice colitis. J. Ethnopharmacol 150 (3), 925-934. doi:10. 1016/j.jep.2013.09.030

Mineta, K., Yamamoto, Y., Yamazaki, Y., Tanaka, H., Tada, Y., Saito, K., et al. (2011). Predicted expansion of the claudin multigene family. FEBS Lett. 585 (4), 606-612. doi:10.1016/j.febslet.2011.01.028

Nuñez-Sánchez, M. A., García-Villalba, R., Monedero-Saiz, T., García-Talavera, N. V., Gómez-Sánchez, M. B., Sánchez-Álvarez, C., et al. (2014). Targeted metabolic profiling of pomegranate polyphenols and urolithins in plasma, urine and colon tissues from colorectal cancer patients. Mol. Nutr. Food Res. 58 (6), 1199-1211. doi:10.1002/mnfr.201300931 
Raleigh, D. R., Marchiando, A. M., Zhang, Y., Shen, L., Sasaki, H., Wang, Y., et al. (2010). Tight junction-associated MARVEL proteins marveld3, tricellulin, and occludin have distinct but overlapping functions. Mol. Biol. Cel. 21 (7), 1200-1213. doi:10.1091/mbc.E09-08-0734

Rosenthal, R., Günzel, D., Piontek, J., Krug, S. M., Ayala-Torres, C., Hempel, C., et al. (2020) Claudin-15 forms a water channel through the tight junction with distinct function compared to claudin-2. Acta Physiol. 228 (1), e13334. doi:10. 1111/apha.13334

Saeed, M., Naveed, M., Bibi, J., Kamboh, A. A., Arain, M. A., Shah, Q. A., et al. (2018). The promising pharmacological effects and therapeutic/medicinal applications of punica granatum L. (Pomegranate) as a functional food in humans and animals. Recent Pat Inflamm. Allergy Drug Discov. 12 (1), 24-38. doi:10.2174/1872213X12666180221154713

Samanta, P., Wang, Y., Fuladi, S., Zou, J., Li, Y., Shen, L., et al. (2018). Molecular determination of claudin-15 organization and channel selectivity. J. Gen. Physiol. 150 (7), 949-968. doi:10.1085/jgp.201711868

Seeram, N. P., Lee, R., and Heber, D. (2004) Bioavailability of ellagic acid in human plasma after consumption of ellagitannins from pomegranate (Punica granatum L.) juice. Clin. Chim. Acta 348 (1-2), 63-68. doi:10.1016/j.cccn. 2004.04.029

Shen, L., Black, E. D., Witkowski, E. D., Lencer, W. I., Guerriero, V., Schneeberger, E. E., et al. (2006). Myosin light chain phosphorylation regulates barrier function by remodeling tight junction structure. J. Cel Sci. 119 (10), 2095-2106. doi:10.1242/jcs.02915

Suzuki, T., Tanabe, S., and Hara, H. (2011). Kaempferol enhances intestinal barrier function through the cytoskeletal association and expression of tight junction proteins in Caco-2 cells. J. Nutr. 141 (1), 87-94. doi:10.3945/jn.110. 125633

Takehara, M., Nishimura, T., Mima, S., Hoshino, T., and Mizushima, T. (2009). Effect of claudin expression on paracellular permeability, migration and invasion of colonic cancer cells. Biol. Pharm. Bull. 32 (5), 825-831. doi:10. 1248/bpb. 32.825

Turner, J. R., Angle, J. M., Black, E. D., Joyal, J. L., Sacks, D. B., and Madara, J. L. (1999). PKC-dependent regulation of transepithelial resistance: roles of MLC and MLC kinase. Am. J. Physiol. 277 (3), C554-C562. doi:10.1152/ajpcell.1999. 277.3.C554
Van Itallie, C. M., Fanning, A. S., and Anderson, J. M. (2003). Reversal of charge selectivity in cation or anion-selective epithelial lines by expression of different claudins. Am. J. Physiol. Ren. Physiol 285 (6), F1078-F1084. doi:10.1152/ ajprenal.00116.2003

Wang, F., Schwarz, B. T., Graham, W. V., Wang, Y., Su, L., Clayburgh, D. R., et al. (2006). IFN-gamma-induced TNFR2 expression is required for TNFdependent intestinal epithelial barrier dysfunction. Gastroenterology. 131 (4), 1153-1163. doi:10.1053/j.gastro.2006.08.022

Ye, D., and Ma, T. Y. (2008). Cellular and molecular mechanisms that mediate basal and tumour necrosis factor-alpha-induced regulation of myosin light chain kinase gene activity. J. Cel Mol Med. 12 (4), 1331-1346. doi:10.1111/j. 1582-4934.2008.00302.x

Yu, A. S., Cheng, M. H., Angelow, S., Günzel, D., Kanzawa, S. A., Schneeberger, E. E., et al. (2009) Molecular basis for cation selectivity in claudin-2-based paracellular pores: Identification of an electrostatic interaction site. J. Gen. Physiol. 133, 111-127. doi:10.1085/jgp.200810154

Zhao, S. S., Ma, D. X., Zhu, Y., Zhao, J. H., Zhang, Y., Chen, J. Q., et al. (2018). Antidiarrheal effect of bioactivity-guided fractions and bioactive components of pomegranate (Punica granatum L.) peels. Neurogastroenterol Motil. 30 (7), e13364. doi:10.1111/nmo.13364

Zolotarevsky, Y., Hecht, G., Koutsouris, A., Gonzalez, D. E., Quan, C., Tom, J., et al. (2002). A membrane-permeant peptide that inhibits MLC kinase restores barrier function in in vitro models of intestinal disease. Gastroenterology 123 (1), 163-172. doi:10.1053/gast.2002.34235

Conflict of Interest: The authors declare that the research was conducted in the absence of any commercial or financial relationships that could be construed as a potential conflict of interest.

Copyright (c) 2021 Hering, Luettig, Jebautzke, Schulzke and Rosenthal. This is an open-access article distributed under the terms of the Creative Commons Attribution License (CC BY). The use, distribution or reproduction in other forums is permitted, provided the original author(s) and the copyright owner(s) are credited and that the original publication in this journal is cited, in accordance with accepted academic practice. No use, distribution or reproduction is permitted which does not comply with these terms. 